\title{
INVASIVE DUCTAL BREAST CARCINOMA: MORPHOLOGICAL FEATURES OF MOLECULAR SUBTYPES
}

\author{
Volos L. I., Dudash A. P.
}

\section{INTRODUCTION}

Breast cancer is the most common cancer with over 2.2 million reported cases in 2020. Breast cancer is the leading cause of cancer death in women. In 2020, 685,000 women died from this disease. On March 8, 2021, on International Women's Day, the World Health Organization (WHO) launched a new Global Breast Cancer Initiative. Its goal is to reduce mortality from this cancer by 2.5 percent annually until 2040 . This will help save the lives of 2.5 million women. The number of healthy life years lost by women with this diagnosis in the world exceeds that of any other type of cancer in women. Breast cancer occurs worldwide in women of all ages after puberty, but the disease increases with age. Most cases and deaths from breast cancer occur in low- and middle-income countries. There are significant differences in rates associated with breast cancer between high-income and low-income countries. The five-year life expectancy for breast cancer exceeds $90 \%$ in high-income countries, but is only $66 \%$ in India and $40 \%$ in South Africa ${ }^{1}$. According to the WHO, published in 2018, the number of deaths from breast cancer in Ukraine amounted to 8,983 people, or $1.49 \%$ of the total number of deaths. With an agespecific mortality rate of 20.93 per 100,000 population, Ukraine ranks 36th in the world. Early diagnosis and treatment of this pathology is an important not only medical, but also a social problem (Bulletin, 2020) ${ }^{2}$.

\section{The problems prerequisites emergence and the problems formulation}

Improvement significant of result of treatment is possible in the case of a combination of early detection and subsequent effective treatment using three methods - surgery, radiation therapy and chemotherapeutic treatment. Although surgery is the main treatment, adjuvant chemoradiotherapy is essential. Up to $40 \%$ of patients with stages I - II have a recurrence of the disease after surgery ${ }^{3,4}$. The development of personalized medicine proposes

1 Рак грудної залози. ВООЗ, 2021. https://www.who.int/news-room/ fact-sheets/detail/breast-cancer

${ }^{2}$ Bulletin of National Cancer Registry of Ukraine (English). (2020).

${ }^{3}$ Siegel R., Ma J., Zou Z., Jemal A. Cancer statistics, 2014.

${ }^{4}$ Strehl, JD, Wachter, DL, Fasching, PA, Beckmann, MW, Hartmann, A. Invasive breast cancer: recognition of molecular subtypes. Breast Care, 2011; 6: 258-64. 
the problem of more precise prediction of the course of the disease, the volume of surgical intervention, and the choice of targeted therapy in patients with breast cancer. In this regard, various molecular genetic biomarkers of cancer are being investigated, such as mutations in the genome of breast cancer patients and the level of expression of genes responsible for the development of the disease. Breast cancer is a heterogeneous disease and implies differences in morphology of tumor types and expression subtypes. Since breast cancer contains a wide range of gene mutations in the genomes, they can also serve as markers in the diagnosis and treatment of tumors. Rapid progression in next generation sequencing technology have resulted in a of mutation data ${ }^{5}$.

The introduction of expression microarray analysis and the rapid progression in data processing technology have greatly contributed to the deepening of researcher's knowledge about molecular abnormalities in cancer cells and today the question is to make the best use of the large amount of accumulated data to search for markers that are useful for oncologists in making a diagnosis and choosing an adequate treatment ${ }^{6}$.

Study of the tumors on the molecular level led to the formation of 2 ways to study the clinical significance of differences in expression profiles. Some studies were aimed at systematizing breast cancer patients that could identify different tumor subtypes, while others were aimed at elucidating specific changes in order to predict the effect of therapy and have prognostic value ${ }^{7}$. Some studies based on microarray analysis have been aimed at identifying parameters such as prognosis of disease recurrence ${ }^{8}$ and tendency to metastasis ${ }^{9}$.

The most relevant direction, which has been developing recently, is devoted to the search for tumor biomarkers that can predict the development and outcome of the disease or the response to therapy, and will also allow creating and supplementing the molecular classification of breast cancer.

5 Meyerson M., Gabriel S., Getz G. Advances in understanding cancer genomes through second-generation sequencing. Nat Rev Genet 2010; 11(10): 685-96.

${ }^{6}$ Pfeifer GP, Hainaut P. Next-generation sequencing: emerging lessons on the origins of human cancer. Curr Opin Oncol 2011; 23(1): 62-8.

${ }^{7}$ Russnes HG, Navin N, Hicks J, Borresen-Dale AL. Insight into the heterogeneity of breast cancer through next-generation sequencing. J Clin Invest 2011; 121(10): 3810-8.

${ }^{8}$ van de Vijver MJ, He YD, van't Veer LJ et al. A gene-expression signature as a predictor of survival in breast cancer. N Engl J Med 2002; 347(25): 1999-2009.

${ }^{9}$ Wang Y, Klijn JG, Zhang Y et al. Gene expression profiles to predict distant metastasis of lymph-node-negative primary breast cancer. Lancet 2005; 365 (9460): 671-9. 


\section{Molecular classification of breast cancer and modern prognostic systems}

At present time, the molecular genetic classification of breast cancer proposed in 2000 by Perou CM et al. is used to determine the appropriate treatment tactics ${ }^{10}$. This approach is based on the expression patterns of native genes, which show a greater difference in expression between tumors than inside the tumor. The molecular subtype identifies subgroups with different biological properties and response to treatment.

Sørlie T. et al. ${ }^{11}$ identified the main subtypes of breast cancer associated with changes in the expression profile of certain genes involved in tumor development. The study allowed the statistical method using cluster hierarchical analysis to identify 4 groups of patients ( $\mathrm{p}<0.001)$ : luminal A subtype, luminal B subtype, basal-like (triple negative) subtype and HER-2 + subtype, each of which has a different prognosis and targets for therapy. ${ }^{12}$ Molecular classification was based on the use of markers ${ }^{13}$ to characterize subtypes:

- luminal A subtype diagnosed up 30-45\% of all breast cancer observations, according to some authors, up to $60 \%$, and mainly in postmenopausal women. These are estrogen-dependent tumors with high ER gene expression, slightly aggressive tumors, negative HER-2/neu expression and low proliferative activity (Ki-67 expression is less than 20\%). For this group, in comparison to the rest, there are low rates of recurrence and high rates of overall survival. This histogenetic group of breast cancer is characterized by high sensitivity to hormone therapy (tamoxifen, aromatase inhibitors).

- Luminal B subtype tumor accounts, according to the authors, for $14-18 \%$ of all observations and is diagnosed in patients of premenopausal age. The tumor is characterized by a positive expression of receptors for estrogen and progesterone, but depending on HER-2/neu amplification and Ki-67 proliferative activity, two types are possible: with Ki-67 expression over $20 \%$ in combination with a negative HER-2/neu status and with a positive expression of HER-2/neu, regardless of Ki-67

${ }^{10}$ Perou CM, Sørlie T, Eisen MB. et al. Molecular portraits of human breast tumours. Nature 2000; 406(6797): 747-52.

11 Sørlie T, Perou CM, Tibshirani R et al. Gene expression patterns of breast carcinomas distinguish tumor subclasses with clinical implications. Proc Natl Acad Sci USA 2001; 98(19): 10869-74.

${ }^{12}$ Wirapati P, Sotiriou C, Kunkel S et al. Meta-analysis of gene expression profiles in breast cancer: toward a unified understanding of breast cancer subtyping and prognosis signatures. Breast Cancer Res 2008;10(4): R65.

13 Nielsen TO, Hsu FD, Jensen $\mathrm{K}$ et al. Immunohistochemical and clinical characterization of the basal-like subtype of invasive breast carcinoma. Clin Cancer Res 2004; 10(16): 5367-74. 
level. In comparison with luminal $\mathrm{A}$, it is more often accompanied by metastatic lesions of the lymph nodes and recurrence. These neoplasms are more often insensitive to chemotherapy and hormone therapy, but are sensitive to transtuzumab in cases with a positive expression of HER-2/neu.

- HER-2/neu positive breast cancer accounts, according to the authors, for $8-15 \%$ of all observations, is characterized by an aggressive course, low overall survival rates, negative expression of receptors for estrogen and progesterone, but pronounced amplification of the HER2 / neu oncogene, a high proliferative index. activity with Ki-67 expression over $20 \%$. The tumors are not sensitive to hormone therapy, but are sensitive to transtuzumab. According to the TNM system, most of them belong to T3 (tumor more than $5 \mathrm{~cm}$ in the largest dimension) and N1-2 tumors.

- The triple negative subtype accounts, according to the authors, for $10-17 \%$ of all observations. These are estrogen-independent aggressive tumors with the ER-, PR-, HER-2- phenotypes and they are diagnosed in young patients. According to research by Lehmann BD. et al. ${ }^{14}$ the immunohistochemically established triple negative cancer profile is heterogeneous and consists of 6 molecular subtypes that show completely different gene ontologies and are characterized by potentially new therapeutic targets. These are two basal classes (BL1 and BL2), immunomodulatory (IM), mesenchymal (M), with mesenchymal stem cells (MSL), and luminal androgen receptor (LAR) class, characterized by AR expression $^{15,16}$.

Triple negative tumors are large, more often metastasize to lymph nodes and distant organs, and are characterized by lower survival rates. Such tumors are sensitive to chemotherapy, including anthracycline and taxanecontaining regimens ${ }^{17}$ and antiandrogen therapy with enzalutamide ${ }^{18}$.

The selection of the considered subtypes has not only classification, but also clinical significance. In particular, estrogen-dependent and HER-2-negative luminal A and B breast cancers predict 10-year survival

${ }^{14}$ Lehmann BD., Jovanović B, Chen X, Estrada MV et al. Refinement of triplenegative breast cancer molecular subtypes: implications for neoadjuvant chemotherapy selection. PLoS One 2016; 11: p.e0157368.

${ }^{15}$ Yadav BS, Chanana P, Jhamb S. Biomarkers in triple negative breast cancer: A review. World J Clin Oncol 2015; 6(6): 252-63.

${ }^{16}$ Ahn SG, Kim SJ, Kim C, Jeong J. Molecular classification of triple-negative breast cancer. J Breast Cancer 2016; 19: p. 223.

${ }^{17}$ Pandy JP, Balolong-Garcia JC, Cruz-Ordinario MB. et al. Triple negative breast cancer and platinum-based systemic treatment: a meta-analysis and systematic review. BMC Cancer 2019; 19: 1065.

${ }^{18}$ Traina TA, Miller K, Yardley DA, Eakle J, Schwartzberg LS, O'Shaughnessy J. et al. Enzalutamide for the treatment of androgen receptor-expressing triple-negative breast cancer. J Clin Oncol 2018; 36: 884-890. 
regardless of systemic therapy. In HER-2 - positive tumors, the subgroups differ in sensitivity to chemoradiotherapy. A triple negative subtype consists of 6 molecular subtypes. The various molecular classes of triple negative subtype of breast cancer have different response to chemotherapy.

A more accurate prognosis of the development of breast cancer can be made using modern prognostic systems. The most important factors determining regional metastasis include the histological form of cancer, the degree of differentiation and the severity of the invasive component of the tumor. In addition, a detailed study of the morphological structure of the tumor in different molecular genetic types of breast cancer is necessary to find the best prediction criteria of the course of the tumor process.

The aim of the study was to establish the morphological heterogeneity of various molecular genetic subtypes of invasive ductal breast cancer.

\section{Basic characteristics of the study groups}

The basis of scientific work was a complex morphological research, including immunohistochemical study of 193 cases of infiltrative ductal breast cancer. The target sample for the study was formed retrospectively from the total number of patients with infiltrative ductal breast cancer. The study was based on information from medical histories and outpatient cards of dispensary observation of patients who underwent specialized antitumor treatment at the Lviv Oncology Regional Medical and Diagnostic Center in 2017. The sample was formed in accordance with the aim and objectives of the research.

The main factors that were taken into account and formed the inclusion threshold were the characteristics of the oncological process, in particular clinically, radiologically (mammographically), ultrasonographically, MRI and morphologically verified infiltrative ductal breast cancer without distant metastases (M0), absence (N0) or presence of regional lymphadenopathy with morphologically confirmed oncological origin, which meets the criteria N1-3, the exclusion of neoadjuvant treatment. The research material was collected in 2017 and respectively to national and international recommendations, the TNM classification of the seventh edition was used. The study included successive cases respectively the above criteria.

The current study did not include patients without histological verification of breast cancer, with a morphological picture of tumor of nonepithelial origin or non-invasive (intraductal cancer in situ) or invasive lobular breast cancer, with recurrence of the tumor process and patients under 18 years of age.

After careful analysis of the history of the disease and the clinical portrait of patients from the study project were excluded profiles of patients who 
underwent preoperative chemoradiation or hormone therapy and previous surgery.

Data on 193 patients with infiltrative ductal breast cancer are contained in the archives of the Lviv Oncological Regional Medical and Diagnostic Center.

The study was approved by the Commission on Bioethics of Danylo Halytsky Lviv National Medical University and conducted in compliance with the basic bioethical provisions of the Council of Europe Convention on Human Rights and Biomedicine (04.04.1997), Helsinki Declaration Medical Association on the ethical principles of conducting scientific medical research with human participation (1964-2008), as well as the order of the Ministry of Health of Ukraine № 690 from 23.09.2009

Research design

After standardization by target age, clinical and histological parameters, a general sample of 193 patients was trichotomized based on the expression status of ER / PR / HER2 / neu and Ki-67 into four molecular subtypes according to consensus definitions of molecular subtypes St. Gallen 2019 and formed the following cohorts: luminal A subtype; luminal B subtype; Her-2/neu positive subtype; triple-negative subtype.

1. Luminal A $(n=79)$ :

- ER and / or PR positive and HER-2/neu negative, Ki-67 < $20 \%$.

2. Люмінальний B $(n=43)$ :

- ER and / or PR positive, HER-2/neu negative, and Ki-67 $\geq 20 \%$ $(n=14)$ or

- ER and / or PR positive and HER-2/neu positive ( $n=29)$

3. HER-2/neu positive (ER and PR negative and HER-2/neu positive) $(\mathrm{n}=39)$

4. Triple-negative (ER, PR and HER-2/neu negative) $(n=32)$.

In our study we used an antibody panel (Dako, Denmark) to determine the expression of ER, PR sex hormones - monoclonal rabbit antibodies to estrogen receptor) (Clone ER1, dilution 1: 1, Dako Flex) and progesterone receptor (Clone PR 636, dilution 1: 1, Dako, Flex).

The Allred Scoring Guideline scale was used to assess the level of ER or PR expression. ${ }^{19}$

The calculated integrative indicator makes it possible to define the case under study into one of four main groups: a group with an expression level of 0 points (complete absence of stained nuclei, index $=0$ ), a group with a weak color level (an index from 2 to 4 points), a group with an average level expression (index from 5 to 6 points) and a group with a high level of

${ }^{19}$ Allred DC, Harvey JM, Berardo M, Clark GM. Prognostic and predictive factors in breast cancer by immunohistochemical analysis. Mod. Path. 1998; 11: 155-168. 
expression (index is from 7 to 8 points). The percentage of tumor cells expressing ER or PR was also indicated. A positive reaction is level ER and / or PR are positive $\geq 1 \%$, however, according to the recommendations, ER values from $1 \%$ to $9 \%$ should be regarded as doubtful (St Gallen Consensus, 2019).

Membrane staining was evaluated for HER-2 / neu (Clone SP3, dilution 1: 1, Thermo scientific) according to Hercep Test TM as follows: when determining one of 4 categories of HER-2/neu expression levels $(0,1+, 2+, 3+)$, we were guided by the updated recommendations of ASCO / CAP, 2013. HER-2/neu status, assessed as 0 and 1+, was considered negative, assessed as $3+$ was positive, at an indeterminate level of HER-2/neu status (assessed as 2+), an additional molecular genetic study (ISH HER2) was carried out to establish the presence or absence of amplification of the HER-2 gene in the cells. The presence of amplification allowed us to speak of a positive HER-2/neu tumor status.

To study the proliferative activity of tumor cells we used rabbit monoclonal antibodies to the protein Ki-67 (Clone MIB-1, dilution 1: 1, Dako, Flex). Ki-67 expression was assessed in the invasive component at the periphery of tumor growth. Only nuclear staining was assessed; all stained nuclei of tumor cells were taken into account, regardless of the color intensity. The Ki-67 score was interpreted according to the 2019 San Gallen Consensus, with a Ki-67 average of $20 \%, 30 \%$ or more was considered high, and $10 \%$ or less was considered low. Immunohistochemical studies were carried out in a certified laboratory "Western Ukrainian Histological Laboratory", Lviv. The control of the IHC study was the use of tissue with a previously established positive and negative reaction and internal control of the reaction of the results.

Each case was reviewed by the authors and histologically classified according to certain characteristics. General morphological data included tumor size, assessment of tumor location in the surgical sample, tumor color, tumor edges and affected lymph nodes. For microscopic examination, tumor tissue, tissue from lines of surgical resection, breast tissue outside the evident tumor, all identified lymph nodes were presented.

Samples of primary tumor tissue after macroscopic examination were fixed in neutral buffered $10 \%$ formalin, carried out the conductance of pieces of biological material in solutions of alcohols of ascending concentration, prepared paraffin blocks. On a Microtome Manual Microm HM325 serial standard sections were made with a thickness of $5 \pm 1 \mu \mathrm{m}$, which were placed on ordinary slides for histological staining or Thermo Scientific ${ }^{\mathrm{TM}}$ SuperFrost Plus ${ }^{\mathrm{TM}}$ adhesion slides for immunohistochemical studies. Histological examination was performed on deparaffined sections of $5 \pm 1$ $\mu \mathrm{m}$, which were stained with hematoxylin and eosin according to standard 
methods. Stained slides were examined under microscope Leica DM 750 (Leica Microsystems GmbH, Germany) to determine the type of tumor, the differentiation grade, the presence of secondary changes such as necrosis, inflammation, sclerosis, peritumoral lymphatic infiltration and invasion.

Tumors were diagnosed according to the WHO classification of breast tumors $^{20,21}$. The characteristics of the parenchymal component of the tumor (formation of various morphological structures, cell polymorphism, mitosis, tumor invasion beyond the basal membrane), the microenvironment of the tumor were evaluated. Tumors were classified according to grade of differentiation based on the classification of the Scarff - Bloom Richardson, modified by Elston and Ellis ${ }^{22}$, which takes into account the ability of neoplasia to form tubular and glandular structures, the degree of nuclear polymorphism and the number of mitoses: G1 - well differentiated tumor, G2 - moderately differentiated tumor and G3 - poorly differentiated tumor. TNM stages were determined according to the 7th edition of the AJCC Cancer Staging Manual $^{23}$.

Histological sections of $5 \pm 1 \mu \mathrm{m}$ were subjected to standard deparaffinization and dehydration in xylene and alcohols in increasing concentrations. After dewaxing and rehydration of the sections, used heatinduced in TEG buffer at $\mathrm{pH} 9.0$ for epitope retrieval and inhibiting the activity of endogenous peroxidase with $3 \%$ hydrogen peroxide solution and applying blocking serum. Incubation with primary antibodies was performed according to the instructions of the manufacturers, visualization of the IHC reaction was performed using the detection system DAKO EnVision + System with diaminobenzidine (Dako). The sections were stained with Mayer's hematoxylin and enclosed in Canadian balm.

Routine microscopy, photographing of micropreparations, evaluation of immunohistochemical staining was performed on a light optical universal laboratory microscope Leica DM 750 (Leica Microsystems GmbH, Germany) with a digital video camera Leica ICC50 HD.

All calculations were performed using the statistical software package Statistica ${ }^{\circledR}$ for Windows 13.0 (StatSoft Inc., license №JPZ804I382130ARCN10-J). The results were represented by the interval

${ }^{20}$ Tavassoli FA. Tumor of the Breast and female genital organs. World Health Organization Classification of Tumours / FA. Tavassoli, P Devilee. Lyon: IARC Press, 2003. 432 p.

${ }^{21}$ Lakhani SR, Ellis IO, Schnitt SJ. WHO Classification of Tumours of the Breast. Lyon, 2012. P. 19-20.

22 Bloom-Richardson (SBR) grading: a pleiotropic marker of chemosensivity in invasive ductal breast carcinomas treated by neoadjuvant chemotherapy / S. Amat, F. Penault-Llorca, H. Cure at al. Int. J. Oncol, 2002; 4: 791-796.

${ }^{23}$ Edge SB, Byrd DR, Compton CC, Fritz AG, Greene FG, Trotti A, eds. AJCC Cancer Staging Manual. 7th ed. New York: Springer; 2010. 
$\mathrm{M} \pm \mathrm{m}$. Significance of differences was assessed by Student's t-test. The difference parameters were considered statistically significant at $p<0,05$.

\section{The morphological characteristics of the analysed tumours}

Among the 193 invasive ductal breast cancer cases, 79 (40,9\%) patients with luminal A subtype, $32(16,6 \%)$ revealed a triple-negative phenotype (TNBC), 39 (20,2\%) patients with HER-2/neu positive (non-luminal) and $43(22,3 \%)$ with Luminal B invasive breast carcinoma.

The 79 patients with luminal A subtype were identified by immunohistochemistry (IHC): ER+, PR+, HER2- and Ki-67 less than 20 percent on surgically resected breast cancer tissue (Fig.1). Nottingham Histologic Grade distribution was as follows: G1 - 10 (12,66\%), G2 $56(70,88 \%)$, and G3 - $13(16,46 \%)$ cases and clinical stage II - $35(44,30 \%)$ and III - $31(39,24 \%)$ was prevailed. The patients with Luminal A subtype had a median age of $60,41 \pm 12,25$ years (range, 32-85 years), 25 (31,6\%) were under 55 years and menopausal status was in $68,4 \%$ of cases. In $42(53 \%)$ cases, the tumors were localized in the left and in $37(47 \%)$ - in the right breast and lymph node metastasis were in 38 cases $(48,10 \%)(\mathrm{p}<0,05)$.

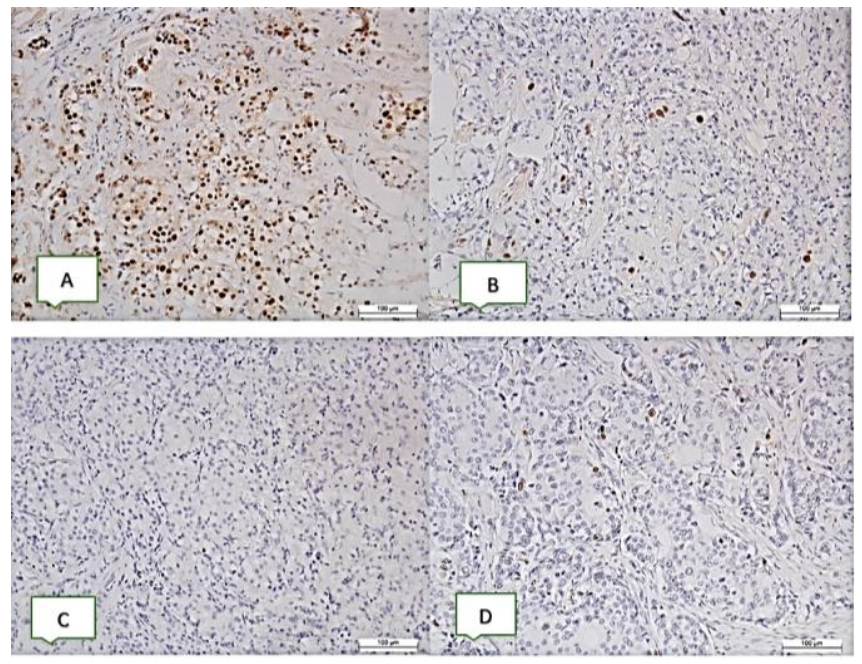

Fig. 1. Invasive ductal carcinoma of the breast. Luminal A subtype. IHC. A - Positive nuclear expression of ER (Clone EP1, Dako Flex). B - Positive nuclear expression of PR (Clone PR 636, Dako, Flex). C - Negative membrane expression of receptors for c-erbB-2 (negative HER-2 / neu status, Clone SP3,

Thermo scientific). D - Positive nuclear expression of Ki-67 in the tissue of invasive carcinoma (Clone MIB-1, dilution 1: 1, Dako, Flex). x200 
Absent expression of ER, PR and HER-2/neu identified TNBC subtype of invasive ductal carcinoma of the breast (Fig.2). The 32 patients with TNBC had a median age of 59 years (range, 31-79 years), $11(34,4 \%)$ were under 55 years. Clinical stage II - $14(43,74 \%)$ and III - $11(34,38 \%)$ was observed and prevailed. Nottingham Histologic Grade distribution was as follows: G1 - 0 (0\%), G2 - 17 (53,13\%), and G3 - 14 (43,74\%), G4 - 1 $(3,13 \%)$ cases. Menopausal status was in $65,6 \%$ of cases. Unilateral lesion of the breast was detected in most cases and tumors were more frequently localized in the outer upper quadrants $(62,5 \%)$.

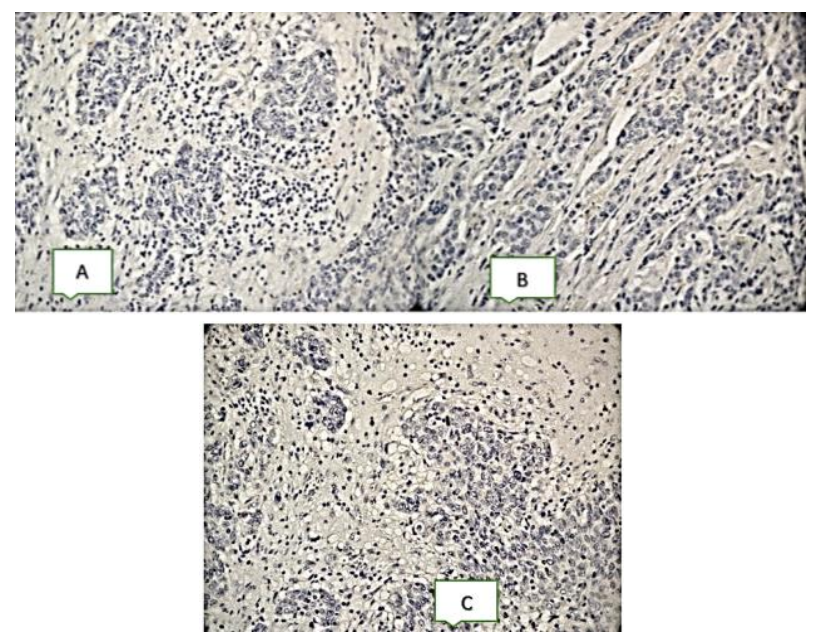

Fig. 2. Invasive ductal carcinoma of the breast, TNBC subtype. IHC.

A - Negative nuclear expression of ER (Clone EP1, Dako Flex). B - Negative nuclear expression of PR (Clone PR 636, Dako, Flex). C - Negative membrane expression of receptors for c-erbB-2 (negative HER-2 / neu status, Clone SP3, Thermo scientific). x200

The HER-2/neu positive (non-luminal) subtype of the invasive ductal breast cancer was recognised by positive HER-2/ neu (3+) or amplified HER2/neu, but in the absence of expression ER and PR (Fig. 3). The median age of the 39 patients with HER-2/neu positive (non-luminal) invasive ductal breast cancer was 50,5 years with range $28-78$ years and $22(56,41 \%)$ were under 55 years, 20 of $39(51,28 \%)$ patients were premenopausal at the time of surgery. In the present study, we identified that tumour size from $2 \mathrm{~cm}$ to $5 \mathrm{~cm}$ was in 23 cases $(58,97 \%)$, tumour grade G1 only in 1 case $(2,56 \%), \mathrm{G} 2$ in 27 cases $(69,23 \%), \mathrm{G} 3$ in 11 cases $(28,21 \%)$ and lymph node metastasis were in 27 cases $(69,23 \%)(\mathrm{p}<0,05)$. 


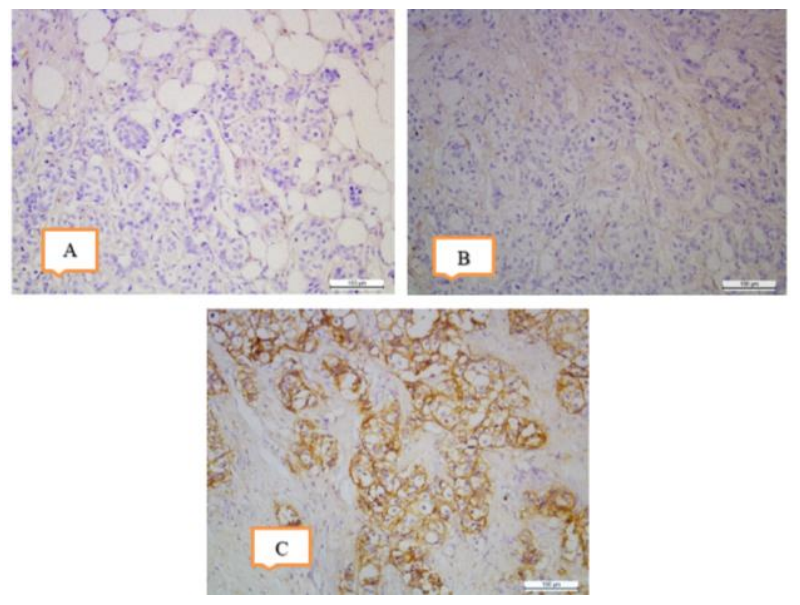

Fig. 3. Invasive ductal carcinoma of the breast, HER-2/neu positive (non-luminal) subtype. IHC. A - Negative nuclear expression of ER (Clone EP1, Dako Flex). B - Negative nuclear expression of PR (Clone PR 636, Dako, Flex). C - Positive membrane expression of receptors for c-erbB-2 (positive HER-2 / neu status, Clone SP3, Thermo scientific). x200

The Luminal B subtype of the invasive ductal carcinoma was divided in two groups. There were luminal B (HER-2/neu negative) and luminal B (HER-2/neu positive) groups. Luminal B (HER-2/neu negative) was recognised by positive ER and/ or PR, negative HER-2 and high Ki-67, and luminal B (HER-2/neu positive) was identified by positive ER and/ or PR, positive HER-2/neu and any level of Ki-67 (Fig.4). The 43 patients with Luminal B invasive ductal breast cancer were divided in luminal B (HER$2 /$ neu negative) and luminal B (HER-2/neu positive) groups. The median age was 49 years (range, $28-70$ years) and 26 patients $(60,47 \%)$ were under 55 years. Clinical stage I - $10(23,26 \%)$, II - $17(39,53 \%)$ and III - $16(37,21 \%)$ was observed. Nottingham Histologic Grade distribution was as follows: G1 - 5 (11,63\%), G2 - 29 (67,44\%), and G3 - 9 (20,93\%) cases. Menopausal status was in 53,49\% of cases.

The morphological study of the infiltrative component of the ductal breast cancer made it possible to reveal a morphological sign that are more characteristic of one or another molecular genetic type of tumour. In the infiltrative component, various morphological structures were diagnosed, such as tubular (Fig. 5 A, B), alveolar (Fig. 5 C, D), trabecular (Fig. 5 F), solid (Fig. 5 E), single tumor cells or isolated tumor cells (Fig. 5 G) and mixed structures (Fig. $5 \mathrm{H}$ ). 
Tubular structures were formed by one or two rows of fairly monomorphic cells with normochromic, sometimes hyperchromic, rounded nuclei (Fig. 5 A, B).

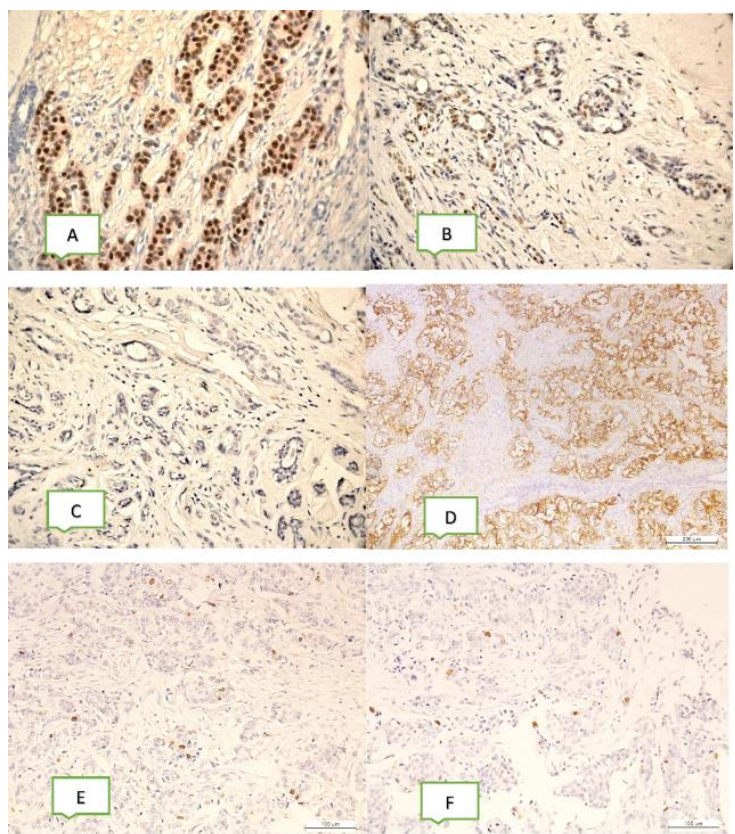

Fig. 4 Invasive ductal carcinoma of the breast, Luminal B subtype. IHC.

A - Positive nuclear expression of ER (Clone EP1, Dako Flex). B - Positive nuclear expression of PR (Clone PR 636, Dako, Flex). C - Negative membrane expression of receptors for c-erbB-2 (negative HER-2 / neu status, Clone SP3, Thermo scientific). D - Positive membrane expression of receptors for c-erbB-2 (positive HER-2 / neu status, Clone SP3, Thermo scientific). x200. E - Positive nuclear expression of $\mathrm{Ki}-67$ more than $20 \%$ in the tissue of invasive carcinoma (Clone MIB-1, dilution 1: 1, Dako, Flex). x200. F - Positive nuclear expression of Ki-67 less than $20 \%$ in the tissue of invasive carcinoma

(Clone MIB-1, dilution 1: 1, Dako, Flex). x200

Alveolar structures were clusters of tumor cells, round or slightly irregular, resembling a round form. The morphology of the cells forming this type of structure ranged from small with moderate cytoplasm and rounded nuclei (Fig. 5D) to large with hyperchromic, irregularly shaped nuclei and moderate cytoplasm (Fig. 5C).

Solid structures were characterized by fields of varying size and shape, which consisted of small cells with moderate cytoplasm and monomorphic nuclei, or large cells with abundant cytoplasm and polymorphic hyperchromic nuclei (Fig. 5E). 
Trabecular structures were short, formed by one row of small, sufficiently monomorphic cells, long or wide, consisting of 2-3 rows of medium-sized cells with moderate cytoplasm, with rounded normochromic or hyperchromic nuclei (Fig. 5F). Individual groups of cells were clusters of two, sometimes 3-4 cells of variable morphology with hyperchromic nuclei (Fig. 5G). But most often, especially in luminal cancer, the infiltrative component had a mixed structure (Fig. 5H).
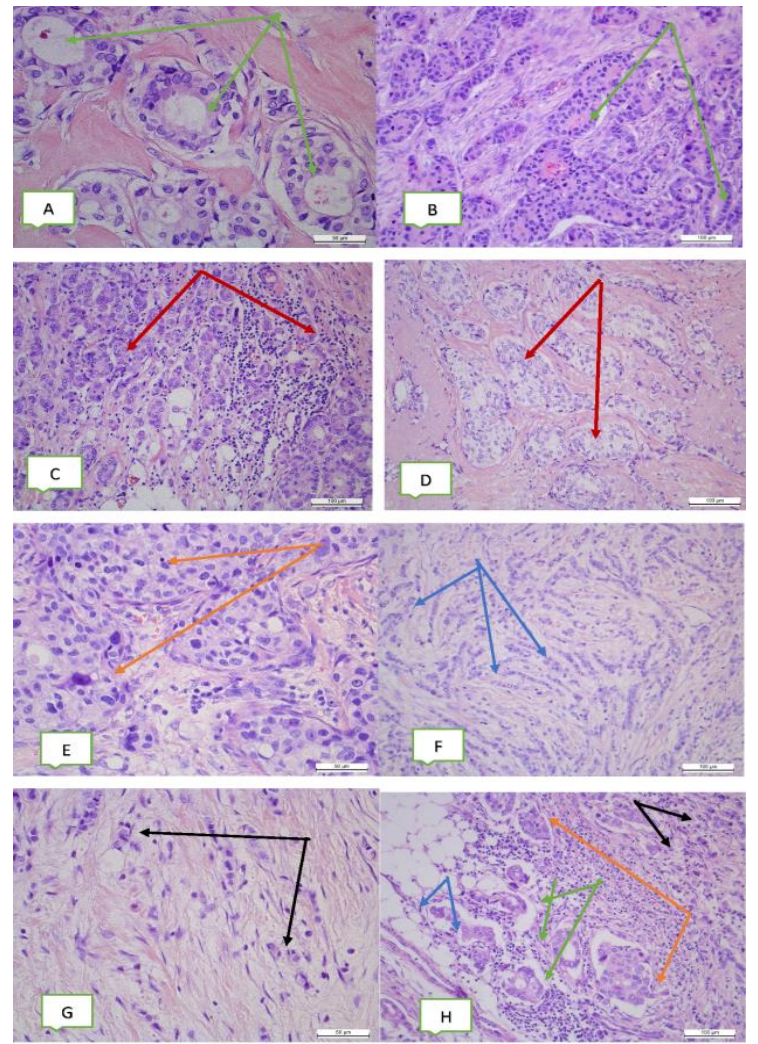

Fig. 5. Infiltrative component of invasive ductal carcinoma of the breast. $A$ and $B$ - Clusters of tumor cells forming typical tubular structures (green arrow). H\&E stain, $A \times 400 ; B \times 200 ; C$ and $D-$ Tumor cells of round or oval shape forming alveolar structures (red arrow). H\&E stain, $\times 200$;

$\mathbf{E}$ - Solid structure of the tumor represented by different size cells with abundant cytoplasm and pleomorphic hyperchromatic nuclei (orange arrow). H\&E stain, $\times 400 ; F$ - Trabecular (blue arrow) structures. H\&E stain, $\times 200$;

G - Individual groups of tumor cells (black arrow). H\&E stain, $\times 400$.

Mixed component, represented different structures. H\&E stain, $H \times 200$ 
The infiltrative component in luminal A and B molecular genetic types was the most diverse: alveolar, trabecular, tubular, solid and separately located groups of tumor cells were diagnosed simultaneously. At the same time, for a triple-negative molecular-genetic type and with overexpression of HER-2/neu, monomorphic structure of tumours was revealed. The infiltrative component consisted of only one of the structure variants was present. There were isolated cases with two variants of structures simultaneously.

\section{CONCLUSIONS}

Thus, we found differences in the morphological structure of the infiltrative component of different molecular genetic subtypes of invasive ductal breast cancer. Luminal A and luminal B breast cancers were characterized by the maximum diversity of the morphological structure of the tumor and the presence of the most differentiated tubular structures. In triple negative type and with overexpression of HER-2 / neu, a monomorphic structure of the infiltrative component with the presence of one type of structures, with a rare presence of tubular structures in it, was observed, which may have an important prognostic value in lymphogenic metastasis.

\section{SUMMARY}

Breast cancer is a heterogeneous disease. Morphological and molecular genetic heterogeneity of the structure of tumor tissue can serve as the basis for differences in the progression of the disease ${ }^{24,25}$

The development of personalized medicine formulates the problem of more accurate prediction of the course of the disease, the volume of surgical intervention, as well as the choice of targeted therapy in patients with breast cancer $^{26}$. In this regard, various molecular genetic biomarkers of cancer are being investigated, such as mutations in the genome of breast cancer patients and the level of expression of genes responsible for the development of the disease.

At present, immunohistochemistry is accepted as adequate method for classification molecular subtypes of breast cancer. Luminal A, luminal B (HER-2/neu positive), luminal B (HER-2/neu negative), HER-2/neu positive and triple negative were diagnosed us according to St. Gallen classification.

${ }^{24}$ Vuong D, Simpson PT, Green B et al. Molecular classification of breast cancer. Virchows Arch 2014; 465(1): 1-14.

${ }^{25}$ Zhao X, Rødland EA, Tibshirani R, Plevritis S. Molecular subtyping for clinically defined breast cancer subgroups. Breast Cancer Res 2015; 17:29.

${ }^{26}$ Prat A, Pineda E, Adamo B et al. Clinical implications of the intrinsic molecular subtypes of breast cancer. Breast 2015; 24 Suppl 2: S. 26-35. 
Luminal A and luminal B breast cancers were characterized by the maximum diversity of the morphological structure of the tumor and the presence of the most differentiated tubular structures. In triple negative type and with overexpression of HER-2 / neu, a monomorphic structure of the infiltrative component with the presence of one type of structures, with a rare presence of tubular structures in it, was observed, which may have an important prognostic value in lymphogenic metastasis.

\section{REFERENCES}

1. Рак грудної залози. ВОО3, 2021. https://www.who.int/newsroom/fact-sheets/detail/breast-cancer.

2. Bulletin of National Cancer Registry of Ukraine (English). Cancer in Ukraine, 2018-2019. Ukrainian cancer registry statistics. 2020; Vol.21 "Cancer in Ukraine", 2018-2019.

3. Siegel R, Ma J, Zou Z, Jemal A. Cancer statistics, 2014. Cancer J Clin 2014; 64 (1): 9-29. DOI: 10.3322/caac.21208. PMID: 24399786.

4. Strehl JD, Wachter DL, Fasching PA, Beckmann MW, Hartmann A. Invasive breast cancer: recognition of molecular subtypes. Breast Care, 2011; 6: 258-64. DOI: 10.1159/000331339.

5. Meyerson M, Gabriel S, Getz G. Advances in understanding cancer genomes through second-generation sequencing. Nat Rev Genet 2010; 11(10): 685-96. DOI: 10.1038/nrg2841. PMID: 2084774.

6. Pfeifer GP, Hainaut P. Next-generation sequencing: emerging lessons on the origins of human cancer. Curr Opin Oncol 2011; 23(1): 62-8. DOI: 10.1097/CCO.0b013e3283414d00. PMID: 21119514.

7. Russnes HG, Navin N, Hicks J, Borresen-Dale AL. Insight into the heterogeneity of breast cancer through next-generation sequencing. J Clin Invest 2011; 121(10): 3810-8. DOI: 10.1172/JCI57088. PMID: 21965338.

8. Van de Vijver MJ, He YD, van't Veer LJ et al. A gene-expression signature as a predictor of survival in breast cancer. N Engl J Med 2002; 347(25): 1999-2009. DOI: 10.1056/NEJMoa021967. PMID: 12490681.

9. Wang Y., Klijn JG, Zhang Y et al. Gene expression profiles to predict distant metastasis of lymph-node-negative primary breast cancer. Lancet 2005; 365 (9460): 671-9. DOI: 10.1016/S0140-6736(05)17947-1. PMID: 15721472 .

10. Perou CM, Sørlie T, Eisen MB. et al. Molecular portraits of human breast tumours. Nature 2000; 406(6797): 747-52. PMID: 10963602.

11. Sørlie T, Perou CM, Tibshirani R et al. Gene expression patterns of breast carcinomas distinguish tumor subclasses with clinical implications. Proc Natl Acad Sci USA 2001; 98(19): 10869-74. DOI: 10.1073/pnas.191367098. PMID: 11553815. 
12. Wirapati P, Sotiriou C, Kunkel S et al. Meta-analysis of gene expression profiles in breast cancer: toward a unified understanding of breast cancer subtyping and prognosis signatures. Breast Cancer Res 2008;10(4): R65. DOI: 10.1186/bcr2124. PMID: 18662380.

13. Nielsen TO, Hsu FD, Jensen $\mathrm{K}$ et al. Immunohistochemical and clinical characterization of the basal-like subtype of invasive breast carcinoma. Clin Cancer Res 2004; 10(16): 5367-74. DOI: 10.1158/ 1078-0432.CCR-04-0220. PMID: 15328174.

14. Lehmann BD, Jovanović B, Chen X, Estrada MV et al. Refinement of triple-negative breast cancer molecular subtypes: implications for neoadjuvant chemotherapy selection. PLoS One 2016; 11: p.e0157368. 10.1371/journal.pone.0157368 CrossRefView Record in ScopusGoogle Scholar.

15. Yadav BS, Chanana P, Jhamb S. Biomarkers in triple negative breast cancer: A review. World J Clin Oncol 2015; 6(6): 252-63. DOI: 10.5306/wjco.v6.i6.252. PMID: 26677438.

16. Ahn SG, Kim SJ, Kim C, Jeong J. Molecular classification of triplenegative breast cancer. J Breast Cancer 2016; 19: p. 223. 10.4048/jbc.2016.19.3.223 CrossRefView Record in ScopusGoogle Scholar.

17. Pandy JP, Balolong-Garcia JC, Cruz-Ordinario MB. et al. Triple negative breast cancer and platinum-based systemic treatment: a metaanalysis and systematic review. BMC Cancer 2019; 19: 1065. https://doi.org/10.1186/s12885-019-6253-5.

18. Traina TA, Miller K, Yardley DA, Eakle J, Schwartzberg LS, O'Shaughnessy J. et al. Enzalutamide for the treatment of androgen receptor-expressing triple-negative breast cancer. J Clin Oncol 2018; 36: 884-890. 10.1200/JCO.2016.71.3495 CrossRefView Record in ScopusGoogle Scholar.

19. Allred DC, Harvey JM, Berardo M, Clark GM. Prognostic and predictive factors in breast cancer by immunohistochemical analysis. Mod. Path. 1998; 11: 155-168.

20. Tavassoli FA. Tumor of the Breast and female genital organs. World Health Organization Classification of Tumours / FA. Tavassoli, P. Devilee. Lyon: IARC Press, 2003. 432 p.

21. Lakhani SR, Ellis IO, Schnitt SJ. WHO Classification of Tumours of the Breast. Lyon, 2012. P. 19-20.

22. Bloom-Richardson (SBR) grading: a pleiotropic marker of chemosensivity in invasive ductal breast carcinomas treated by neoadjuvant chemotherapy / S. Amat, F. Penault-Llorca, H. Cure at al. Int. J. Oncol, 2002; 4: 791-796.

23. Edge SB, Byrd DR, Compton CC, Fritz AG, Greene FG, Trotti A, eds. AJCC Cancer Staging Manual. 7th ed. New York: Springer; 2010. 
24. Vuong D, Simpson PT, Green B et al. Molecular classification of breast cancer. Virchows Arch 2014; 465(1): 1-14. DOI: 10.1007/s00428014-1593-7. PMID: 24878755.

25. Zhao X, Rødland EA, Tibshirani R, Plevritis S. Molecular subtyping for clinically defined breast cancer subgroups. Breast Cancer Res 2015; 17:29. DOI: 10.1186/s13058-015-0520-4. PMID: 25849221.

26. Prat A, Pineda E, Adamo B et al. Clinical implications of the intrinsic molecular subtypes of breast cancer. Breast 2015; 24 Suppl 2: S26-35. DOI: 10.1016/j.breast.2015.07.008. PMID: 26253814.

\section{Information about the authors:} Volos Liliya Ivanivna,

Doctor of Medical Sciences, Professor, Professor at the Department of Pathological Anatomy and Forensic Medicine Danylo Halytsky Lviv National Medical University 69, Pekarska str., Lviv, 79010, Ukraine

Dudash Andrii Petrovych, Postgraduate Student at the Department of Pathological Anatomy and Forensic Medicine Danylo Halytsky Lviv National Medical University 69, Pekarska str., Lviv, 79010, Ukraine Pathologist Western Ukrainian Histological Laboratory 77, Heroiv UPA str., Lviv, 79015, Ukraine 